\title{
Estimación de la estabilidad de un sazonador a base de amaranto reventado y maní tostado, a partir del índice de peróxidos Ing. María Augusta Rosero ${ }^{1}$, Ing. Elena Villacrés. M.Sc. ${ }^{2}$, Dr. Juan Bravo, Ph.D. ${ }^{1}$
}

\section{Resumen}

El objetivo del presente trabajo fue evaluar la estabilidad, durante el almacenamiento, de un sazonador nutritivo elaborado con amaranto reventado y maní tostado, empleando el índice de peróxidos como un indicador del deterioro del producto. Para lo cual se almacenó el sazonador, empacado en fundas de polipropileno y aluminizadas, a condiciones ambientales $\left(17^{\circ} \mathrm{C}\right.$, $50 \% H R)$ y aceleradas $\left(35^{\circ} \mathrm{C}, 90 \% H R\right)$ durante 30 días; se analizó el índice de peróxidos cada 5 días. Se consideró que el índice de peróxidos, sigue una cinética de orden cero durante el tiempo de almacenamiento. Considerando que las grasas y aceites comestibles, pueden alcanzar un valor máximo de índice de peróxidos de $20 \mathrm{mEq} / \mathrm{kg}$, se determinó que el sazonador nutritivo empacado en funda de polipropileno, y almacenado a condiciones ambientales $\left(17^{\circ} \mathrm{C}\right.$ y $\left.50 \% H R\right)$ se mantuvo estable durante 81 días; mientras que el almacenado a condiciones aceleradas $\left(35^{\circ} \mathrm{C}, 90 \% H R\right)$ alcanzó los 68 días. Asimismo el sazonador empaquetado en funda aluminizada, y almacenado a condiciones ambientales alcanzó un tiempo de 101 días; y a condiciones aceleradas, 78 días.

\section{Palabras clave}

Peróxidos, estabilidad, sazonador, amaranto, maní.

\section{Abstract}

The aim of this study was to evaluate the stability during storage of a nutritious seasoning made from popped amaranth and roasted peanuts, using peroxide index as an indicator of deterioration. The seasoning was stored in polypropylene and aluminized bags at room temperature $\left(17{ }^{\circ} \mathrm{C}, 50 \% \mathrm{RH}\right)$ or accelerated conditions $\left(35^{\circ} \mathrm{C}, 90 \% \mathrm{RH}\right)$ during 30 days. The peroxide index was analyzed every five days. It was considered that the peroxide index followed a zero-order kinetic during the storage time. Assuming that oils and fats can reach a maximum level of the peroxide of $20 \mathrm{mEq} / \mathrm{kg}$, it was observed that the nutritional seasonings packaged in the polypropylene bag stored at room temperature or accelerated conditions remained stable for 81 days 68 days, respectively. In the same way, the samples packaged in the aluminized bags and stored at room temperature remained stable for 101 days whereas the samples in the accelerated conditions reached 78 days.

\section{Keywords}

Peroxide, stability, seasoning, amaranth, peanuts.

\footnotetext{
${ }^{1}$ Universidad Tecnológica Equinoccial, Facultad de Ciencias de la Ingeniería, Quito - Ecuador (bvje2633@ute.edu.ec)

2 INIAP- Departamento de Nutrición y Calidad de Alimentos. +593 23007134 (elena.villacres@inap.gob.ec)
} 


\section{Introducción}

De acuerdo a la norma ecuatoriana NTE INEN 2532:2010, los sazonadores, condimentos, adobos o aliños son productos constituidos por una o más especias u oleorresinas de especias, mezcladas con otras sustancias alimenticias, para mejorar y realzar el sabor, color y aroma de los alimentos (INEN, 2010).

Las especias como hojas, semillas, bulbos, flores y frutos se someten a procesos de secado y molienda para ser conservados en polvo. La vida útil de las especias molidas aumenta en ausencia de aire, a una humedad relativa del $60 \%$ como máximo y a una temperatura de $20{ }^{\circ} \mathrm{C}$, ya que bajo condiciones extremas pierden rápidamente su aroma (Astiasarán \& Martínez, 2003).

Según Velásquez (2006), los alimentos con un alto contenido de grasa al interactuar con el oxígeno del ambiente, sufren una alteración denominada rancidez, la misma que causa olores, sabores desagradables y toxicidad si se consume en grandes cantidades.

El Departamento de Nutrición y Calidad, en la Estación Experimental Santa Catalina (INIAP), ejecutó un proyecto destinado a rescatar e incentivar el consumo de amaranto en el Ecuador, y uno de las alternativas fue desarrollar un sazonador a base de amaranto reventado y maní tostado, por lo que en este trabajo se evaluó la estabilidad en el almacenamiento del sazonador, empacado en dos empaques diferentes y a dos condiciones de almacenamiento, analizando el índice de peróxidos.

\section{Materiales y métodos}

Se utilizó amaranto de la variedad INIAP-Alegría y maní de la marca Mascorona. El amaranto fue proporcionado por el Instituto Nacional de Investigaciones Agropecuarias (INIAP); y, el maní, la cebolla, el ajo, el ají, el pimentón, el cilantro, el orégano, el comino, y la sal fueron adquiridos en el mercado local.

El sazonador fue elaborado con un 44\% de amaranto reventado, $44 \%$ de maní tostado y $12 \%$ de especias, todos los ingredientes se mezclaron manualmente (Rosero, 2012).

Para la determinación del índice de peróxidos del sazonador, se siguió el procedimiento descrito por Madrid, et al. (1997).

Para evaluar la estabilidad durante el almacenamiento, se asumió que la cinética del deterioro de la grasa (rancidez) en el sazonador, se ajustaba a una reacción de orden cero, (Casp y Abril, 2003), por lo que se utilizó la ecuación 1.

$$
y=m x+b
$$

Donde:

$$
\begin{aligned}
& \text { y= Límite crítico para el índice de peróxidos }(20 \mathrm{meq} / \mathrm{kg} \text { ) } \\
& \mathrm{x}=\text { Tiempo en el que el alimento alcanza el límite crítico } \\
& \text { m= Pendiente de la recta } \\
& \text { b= Ordenada al origen (punto donde la recta corta el eje y) }
\end{aligned}
$$




\section{Resultados y discusión}

Aplicada la metodología descrita para la elaboración del sazonador y el correspondiente almacenamiento, se obtuvieron los siguientes resultados. La evaluación de los índices de peróxidos del sazonador, almacenado a las dos condiciones se presenta en la Tabla 1.

Tabla 1. Índice de peróxidos (meq/kg) del sazonador, empacado en dos materiales y durante el almacenamiento a condiciones aceleradas y ambientales

\begin{tabular}{|c|c|c|c|c|}
\cline { 2 - 5 } \multicolumn{1}{c|}{} & \multicolumn{2}{|c|}{$\begin{array}{c}\text { Condiciones Aceleradas } \\
\left(35^{\circ} \mathrm{C} \text { y } 90 \% \mathrm{HR}\right)\end{array}$} & \multicolumn{2}{c|}{$\begin{array}{c}\text { Condiciones Ambientales } \\
\left(17^{\circ} \mathrm{C} \text { y } 50 \% \mathrm{HR}\right)\end{array}$} \\
\cline { 2 - 5 } Día & Polipropileno & Aluminizada & Polipropileno & Aluminizada \\
\hline $\mathbf{0}$ & $9.601 \pm 0.378$ & $9.601 \pm 0.378$ & $9.601 \pm 0.378$ & $9.601 \pm 0.378$ \\
\hline $\mathbf{5}$ & $10.925 \pm 0.335$ & $10.808 \pm 0.337$ & $10.274 \pm 0.931$ & $10.518 \pm 0.771$ \\
\hline $\mathbf{1 5}$ & $12.250 \pm 0.124$ & $12.091 \pm 0.406$ & $11.456 \pm 0.574$ & $11.391 \pm 0.081$ \\
\hline $\mathbf{2 0}$ & $12.660 \pm 0.285$ & $12.503 \pm 0.291$ & $11.805 \pm 0.505$ & $11.823 \pm 0.137$ \\
\hline $\mathbf{2 5}$ & $13.147 \pm 0.519$ & $12.737 \pm 1.210$ & $12.682 \pm 0.372$ & $11.971 \pm 1.617$ \\
\hline $\mathbf{3 0}$ & $14.636 \pm 0.112$ & $13.883 \pm 0.088$ & $13.695 \pm 0.699$ & $13.032 \pm 0.045$ \\
\hline
\end{tabular}

La Figura 1 representa la variación del índice de peróxidos del sazonador empacado en funda de polipropileno en condiciones ambientales $\left(17^{\circ} \mathrm{C}\right.$ y $\left.50 \% \mathrm{HR}\right)$, y la Figura 2 la del producto empacado en funda aluminizada en las mismas condiciones por un período de 30 días. Aplicando la ecuación 1 a los datos obtenidos, se obtuvo un coeficiente de correlación superior al 96\% y los siguientes tiempos de almacenamiento hasta alcanzar el límite permitido de índice de peróxidos:

Para el sazonador nutritivo empacado en funda de polipropileno y en condiciones ambientales $\left(17^{\circ} \mathrm{C}\right.$ y $\left.50 \% \mathrm{HR}\right)$, alcanzó un tiempo de estabilidad de 81 días. En cambio, en el empacado en funda aluminizada, se observa que el índice de peróxido aumenta proporcionalmente con el tiempo, alcanzado el límite crítico (20 meq $/ \mathrm{kg}$ ) a los 101 días de almacenamiento, a partir de los cuales no se garantiza su seguridad para el consumo. Este período de tiempo es superior por 20 días, con respecto al tiempo de almacenamiento en el empaque de polipropileno, y un incremento del índice de peróxidos del 36 \%, con respecto al producto recién procesado. 


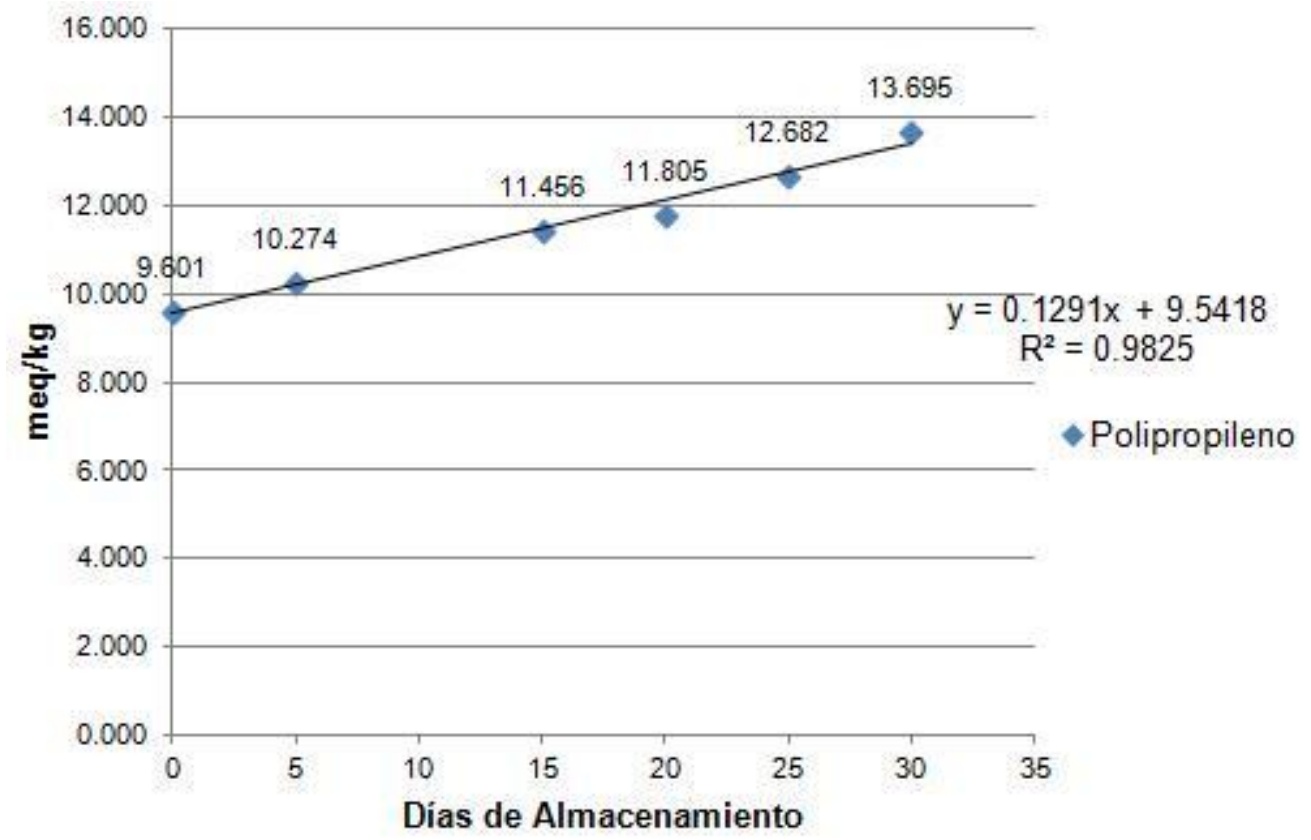

Figura 1. Variación del índice de peróxidos del sazonador, empacado en funda de polipropileno y almacenado en condiciones ambientales $\left(17^{\circ} \mathrm{C}\right.$ y $\left.50 \% \mathrm{HR}\right)$

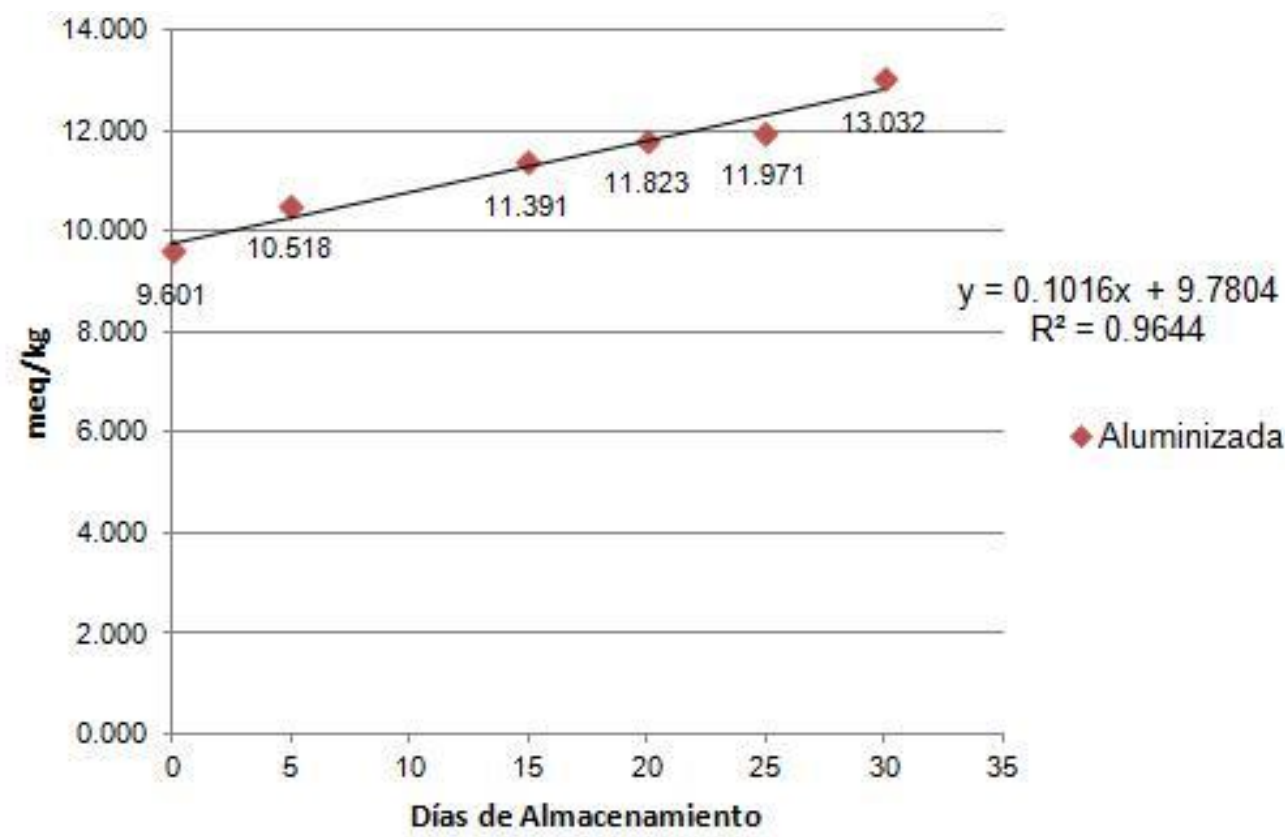

Figura 2. Variación del índice de peróxidos del sazonador, empacado en funda aluminizada y almacenado en condiciones ambientales $\left(17^{\circ} \mathrm{C}\right.$ y $\left.50 \% \mathrm{HR}\right)$ 
Para el sazonador empacado en funda de polipropileno en condiciones aceleradas $\left(35{ }^{\circ} \mathrm{C}\right.$ y $90 \% \mathrm{HR}$ ), los valores obtenidos del índice de peróxidos se presenta en la Figura 3, el coeficiente de correlación de la ecuación 1 es del 97\%, con lo que el valor crítico de peróxidos se alcanza a los 68 días. En cambio en la Figura 4 se aprecia el comportamiento del producto envasado en funda aluminizada, alcanzó en un tiempo de 78 días.

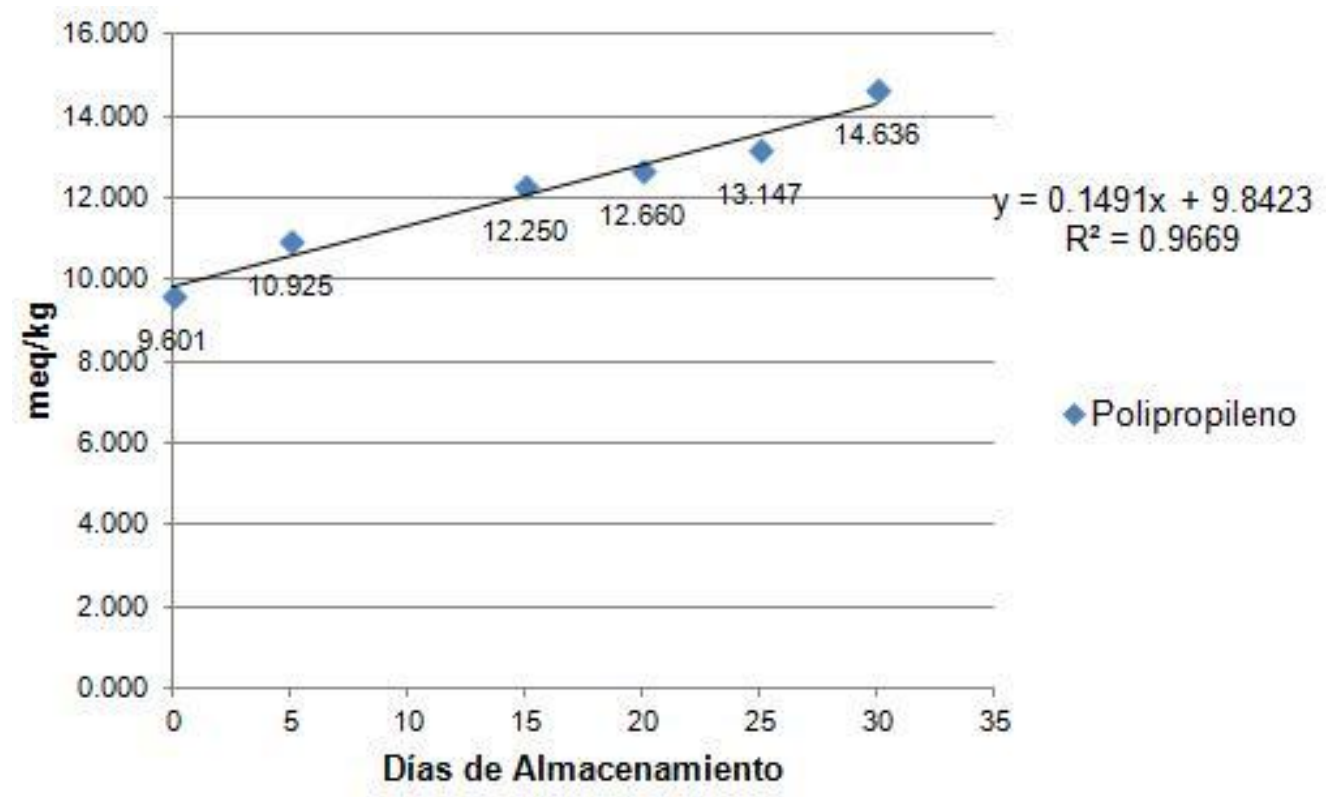

Figura 3. Variación del índice de peróxidos del sazonador, empacado en funda de polipropileno y almacenado en condiciones aceleradas $\left(35^{\circ} \mathrm{C}\right.$ y $\left.90 \% \mathrm{HR}\right)$

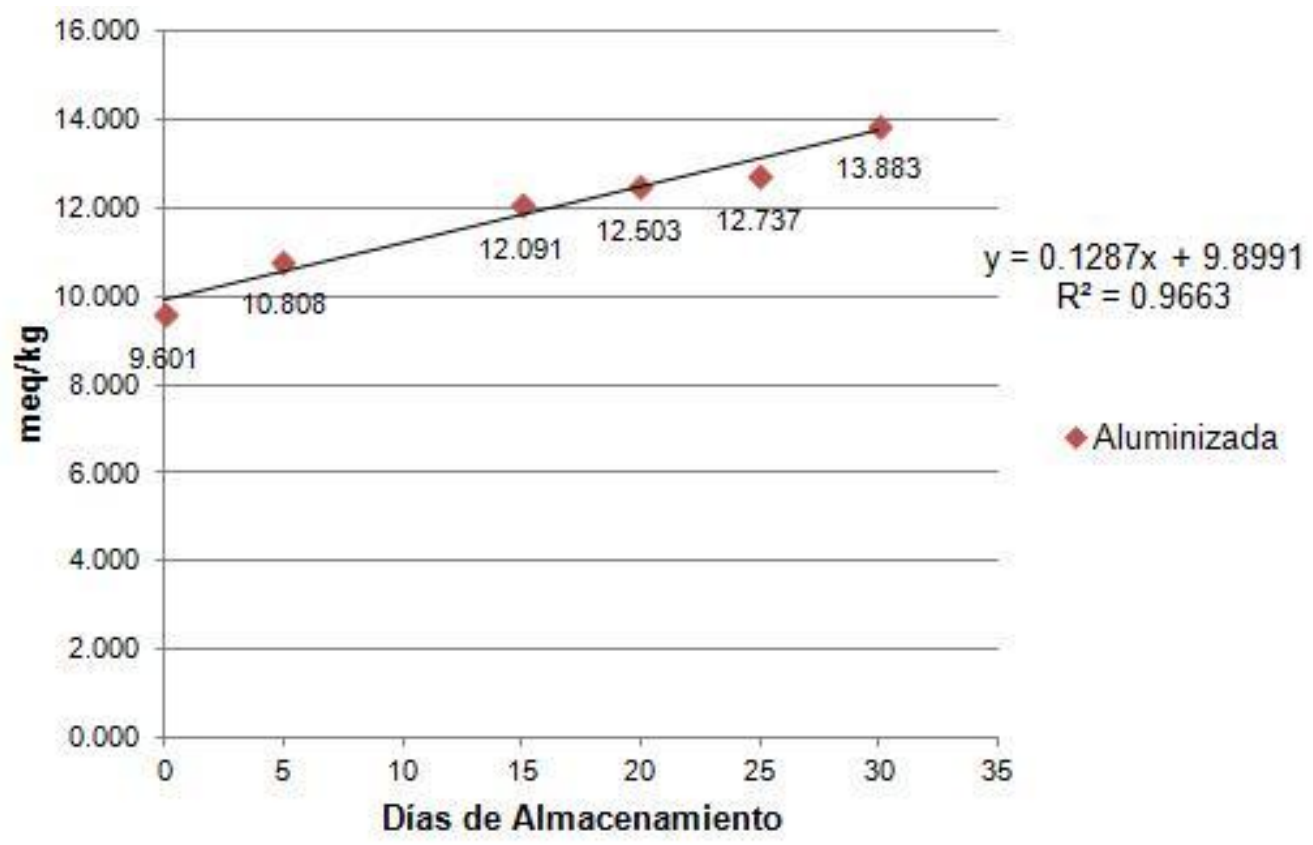

Figura 4. Variación del índice de peróxido del sazonador empacado en funda aluminizada y almacenado en condiciones aceleradas $\left(35^{\circ} \mathrm{C}\right.$ y $\left.90 \% \mathrm{HR}\right)$ 
Comparando el período de estabilidad de este sazonador nutritivo, empacado en funda aluminizada, con los condimentos comerciales, este presenta un tiempo de 101 días, que es la tercera parte de aquellos; esto principalmente puede ser por la presencia de aditivos antioxidantes en la formulación.

\section{Conclusiones y recomendaciones}

El sazonador envasado en un empaque aluminizado, y almacenado a condiciones ambientales $\left(17^{\circ} \mathrm{C}\right.$ y $\left.50 \% \mathrm{HR}\right)$ y aceleradas $\left(35^{\circ} \mathrm{C}\right.$ y $\left.90 \% \mathrm{HR}\right)$, presenta un período de estabilidad mayor respecto al envasado en un empaque de polipropileno.

Se recomienda evaluar la utilización de antioxidantes químicos para incrementar la estabilidad del sazonador.

\section{Bibliografía}

AOCS Monograph Committee. (1990). Edible Fats and Oils Processing: Basic Principles and Modern Practices. Maastricht: American Oil Chemists Society.

Asociación Mexicana del Amaranto. (2003). Amarantum. Recuperado el 7 de 10 de 2011, de Asociación Mexicana del Amaranto: http://www.amaranto.com.mx/salud/ beneficios/beneficios.htm

Astiasarán, I., \& Martínez, A. (2003). Alimentos. Composición y Propiedades. Mexico: McGrawHill.

Casp, A., \& Abril, J. (2003). Procesos de Conservación de Alimentos. Madrid: Mundi-Prensa.

INEN. (2010). Norma Técnica Ecuatoriana NTE INEN 2532:2010. Especias y condimentos. Requisitos.

Instituto Nacional Autónomo de Investigaciones Agropecuarias (INIAP). (2008). Informe Nacional sobre los Recursos Fitogenéticos para la Agricultura y la Alimentación. Quito: INIAP.

Madrid, A., Cenzano, I., \& Vicente, J. (1997). Manual de Aceites y Grasas Comestibles. Madrid: Mundi - Prensa.

Rosero, M. A. (2012). Desarrollo de un sazonador nutritivo a base de amaranto reventado y maní tostado. Trabajo de Titulación de Ingeniería de Alimentos. UniversidadTecnológica Equinoccial. Ecuador.

Ucodep. (24 de 06 de 2011). Quinua, Amaranto, Melloco y Chocho. Un regalo Andino para el mundo. Recuperado el 29 de 09 de 2011, de INIAP: http://www.iniap.gob.ec/

UNIFEM. (1998). Técnicas de Envasado y Empaque. Lima: Asociación Gráfica Educativa.

Velásquez, G. (2006). Fundamentos de Alimentación Saludable. Antioquia: Universidad de Antioquia. 